\title{
Dispersion Effects in the Falkner-Skan Problem and in the Kinetic Theory
}

\author{
Oleg Galaev, Evelina Prozorova \\ Mathematics \& Mechanics Faculty, St. Petersburg State University, Peterhof, Russia \\ Email: e.prozorova@spbu.ru
}

How to cite this paper: Galaev, O. and Prozorova, E. (2017) Dispersion Effects in the Falkner-Skan Problem and in the Kinetic Theory. Journal of Applied Mathematics and Physics, 5, 522-537. https://doi.org/10.4236/jamp.2017.52045

Received: November 23, 2016

Accepted: February 25, 2017

Published: February 28, 2017

Copyright $\odot 2017$ by authors and Scientific Research Publishing Inc. This work is licensed under the Creative Commons Attribution International License (CC BY 4.0).

http://creativecommons.org/licenses/by/4.0/ (c) (i) Open Access

\begin{abstract}
The conservation laws of continuum mechanics and of the kinetic theory with the influence of the angular momentum and associated with its rotation of the elementary volume are considered, the variant of accounting lag is investigated for discrete environment. The analysis of the recording of the Lagrangian function for the collective interaction of the particles with the change of the center of inertia of the moving particles and the effect influence of the angular momentum were used. The equations for gas are calculated from the modified Boltzmann equation and the phenomenological theory. For a rigid body the equations were used of the phenomenological theory, but their interpretation was changed. The nonsymmetric stress tensor was obtained. The Boltzmann equation is written with an additional summand. This situation is typical for discrete environment as the transition from discrete to continuous environment is a key to the issue of mechanics. Summary records of all effects lead to a cumbersome system of equations and therefore require the selection of main effects in a particular situation. The Hilbert paradox was being solved. The simplest problem of the boundary layer continuum (the Falkner-Skan task) and the kinetic theory are discussed. A draw attention at the delay process would be suggested for the description of discrete environment. Results are received for some special cases.
\end{abstract}

\section{Keywords}

Angular Momentum Conservation Laws, Unbalanced Stress Tensor, The Boltzmann Equation, Delay

\section{Introduction}

Our aim is to show that the proposed system of equations can be derived from the first principles, and in this case, at least part of the known properties, which are presented in the textbooks and observed in experiments for turbulent flows, 
can be explained without additional assumptions about the form of the turbulent viscosity, and that one can restrict the molecular viscosity. The basis of the continuum mechanics was built on the laws of conservation of mass, momentum and energy. The fundamental laws of conservation, in addition, are the law of conservation of angular momentum. The conservation laws are obtained by writing the balance equations for the volume element, located in the infinite space. For every conservation law, we have its own chosen elementary volume. However, the law of conservation of angular momentum requires a particular frame of reference, a particular radius vector from the origin to the elementary volume. In the classical approach the law of conservation of angular momentum is not followed. In the mechanics, consideration is made equally of the Lagrangian function for no interacting and collectively interacting particles that are in doubt, especially for the metal and for the ionic bonds. In the classical continuum, mechanics had an opinion on the relatively small contribution of surface forces or as their action has a volume character. However, for long bodies and large gradients of parameters, contribution is significant and can be a cause of instability, leading to changes in the flow. Angular momentum gives emergence of additional forces that can play the role of small perturbations affecting the stability of the structure or destruction of the body. The resulting effects may affect in a critical and a near critical modes of aircraft, rockets, various devices, structures, as well as in some of the natural processes. The value of the additional force is determined by the gradient of physical quantities (density, velocity, momentum). The action of angular momentum, i.e. torque depends substantially on the axis of inertia (center of mass). A volume element can be important due to both its rotation about its center of inertia and involvement of angular momentum, i.e. torque depends substantially on the axis of inertia (center of mass). The proposed new accounting delay interpretation consists in to consideration the difference between the time derivative as a limit of infinity small and end values of the mean free path in a rarefied gas. The role of individual time delay for each particle velocity and average time are debated. The Boltzmann equation is written with an additional summand. This situation is typical for the discrete environment. The transition from discrete to continuous environment is a key in mechanics. Summary of all effects leads to a cumbersome system of equations and therefore requires the selection real situation. There were previously obtained modified energy equation of motion, continuity and momentum for particles without structure, taking into account effects of the change of the angular momentum in the elementary volume. They were resulting from modified Boltzmann equation, which was obtained from the modified Liouville equation. It was built with modified the Lagrangian function. The classical phenomenological theory was used for a solid body but interpretation was varied in this case [1] [2] [3]. Classical ideas are obtained from consideration the volume as exclusive. Now for consideration of the angular momentum the theory of brothers E., Cosserat, F. Cosserat and their modifications are used. Their theory contains additional constant with dimension of length that determined from ex- 
periments [4]. Even classic computation of solution presents considerable difficulties [5]. In [1] [2] [3] influence of the angular momentum on the equations of continuum mechanics was obtained. The proposed theoretical method of consideration for the angular momentum without new empirical constants was suggested. Method is based on the fact that we not have new dimensions to the angular momentum with respect to position and momentum entering into the equation in the classic version. Using conditions of equilibrium of forces leads to a symmetric stress tensor and a violation of "continuity" of the environment that a rigorous analysis requires additional conditions. Another approach proposed in [6]. It should be noted that in general case, ergodicity is not observed, that is very important. Analysis of the non-observance was made by T. G. Elizarova [7]. In conservation laws for space coordinates averaging is fulfilled but about times is not. We have laws that are not symmetric relatively time and space. It is correct when integral method for construction of continuum mechanics function is used for formulation of conservation laws. It is interesting that constructing equations through delta-function gives us the formulation of conservation laws too. Probably, more correct representation for the kinetic theory would be formulation of integral equations that should have average time among molecules collisions. As we spoke higher conditions of forces equilibrium for the construction of continuum mechanics are used. In general mechanics we used the laws of force and angular momentum equilibrium. The laws provide a balance of power conservation of mass, momentum and energy. The fundamental conservation law in addition is the law of conservation of angular momentum. It should be noted that for the kinetic theory (the Boltzmann equation) the law of conservation of angular momentum does not hold. Macroscopic parameters are determined in the equilibrium function of the Chapman-Enskog distribution function in which used the Euler equations parameters and tensor $\boldsymbol{P}$ is symmetric. Besides for the Chapman-Enskog distribution function formally we have values (density, linear moment and energy) with the first-order error. This fact was noted by Hilbert without further use and correction [8] [9] [10] [11]. This is the Hilbert paradox.

$$
\int \varphi(\xi) f^{0} \mathrm{~d} \xi=\int \varphi(\xi) f \mathrm{~d} \xi=\beta,
$$

Here $\beta$-macroscopic parameter, $\varphi(\xi)$-function.

$$
\begin{gathered}
n(t, \boldsymbol{x})=\int f(t, \boldsymbol{x}, \boldsymbol{\xi}) \mathrm{d} \boldsymbol{\xi}, \boldsymbol{u}=\frac{1}{n} \int \xi f(t, \boldsymbol{x}, \boldsymbol{\xi}) \mathrm{d} \boldsymbol{\xi}, \\
P_{i j}=m \int c_{i} c_{j} f(t, \boldsymbol{x}, \boldsymbol{\xi}) \mathrm{d} \boldsymbol{\xi}, q_{i}=\frac{m}{2} \int c^{2} c_{i} f(t, \boldsymbol{x}, \boldsymbol{\xi}) \mathrm{d} \boldsymbol{\xi} .
\end{gathered}
$$

So for the equilibrium in collision integral we have

$$
f(t, \boldsymbol{x}, \boldsymbol{\xi}) \equiv f^{0}(t, \boldsymbol{x}, \boldsymbol{\xi})=n\left\{-\frac{m}{2 k T} c^{2}\right\}, c^{2}=c_{1}^{2}+c_{2}^{2}+c_{3}^{2}=(\boldsymbol{\xi}-\boldsymbol{u})^{2}
$$

and for non-equilibrium distribution function 


$$
f=f^{0}\left[1+\frac{p_{i j} m}{2 p k T} c_{i} c_{j}-\frac{q_{i} m}{p k T} c_{i}\left(1-\frac{m c^{2}}{5 k T}\right)\right]
$$

we have the same macroparameters in $f^{0}$.

There $f$ is distribution function, $n, \boldsymbol{u}, P_{i j}, T$ are macroparameters, $t, \boldsymbol{x}, \boldsymbol{\xi}$ coordinates.

The Navier-Stokes equations of the boundary layer are non-linearity and dissipation interact with each other summand. Non-linearity causes distortion of the original signal. The dissipation reduces the amplitude of the signal. However, we know that in addition to the above factors for a number of tasks are important dispersion effects, i.e. splitting the signal into individual harmonics. Classical equation, which is characterized by the presence and interaction of nonlinearity, dissipation and dispersion, is the Korteweg-de Vries-Burgers equation. Waveform is changing. If in the equations of motion of the system of Navier-Stokes equations we introduce an additional summand with the third derivative, then it will turn into the Korteweg-de Vries-Burgers equation. Usually we suggested that non-linearity and dissipation for large gradients of laminar flows can change flows to turbulent flows. In the Reynolds model actually stand fast and slow variables. In the resulting averaged equations establish the connection between pressure and the Reynolds-averaged flow parameters, but have not the answer to the question of the form of the closing ratio. The process of building relationships based or on empirical evidence or written out of the equation for higher order moments, such as turbulent (fluctuating) kinetic energy. In these equations include new unknowns, and the process is repeated for the specified circuit scenario. For the inertial flow in the equilibrium case, a well-known law of N.A. Kolmogorov's theory of the dimension is performed. At the heart of all theories are the Navier-Stokes equations. Even if involved the Boltzmann equation in order to obtain from it the equations of turbulent flow, the output method is focused on validation of the model Reynolds, built on the basis of the Navier-Stokes equations. It is hardly possible to derive the equation of continuity for the tube of flow for turbulent flow. The main directions of current research include [12] [13] [14] [15] [16]:

1) The increasing complexity of the structure of the dynamic viscosity coefficient

2) The increasing complexity of the equations (the introduction of the summand with the third derivative, a third-order tensor, etc.)

3) The wording of the new system of equations for the functions of the new system

4) Introduction in the equation of the motion of random or accidental forces viscosity

5) Solution of the full three-dimensional unsteady Navier-Stokes equations on detailed grids with high-order schemes

6) Isolation of large eddies with the addition of the flow pattern inside the grid cell by further study the model selected inside the cell

Before proceeding to the solution of specific problems, we present a quote 
from the Loitsyanskii book [12]: "The current lines of pulsating movement of cross streamlines of the mean flow, penetrate from one layer to another, and create the stirring - it is called a molar or turbulent mixing-accompanied by the transport through the boundary between the layers of momentum, energy, heat, and other mechanical or thermodynamic parameters of the mean flow liquid. Given for the turbulent velocity profile are averaged. As the theory of stability in the areas of origin of the turbulence observed first regular vibrational structure." Log infinite plate profiles and tubes, generally obtained final product length, though very elongated. The theoretical profile is defined by introducing the Reynolds stress tensor $\frac{\mathrm{d}^{2} \boldsymbol{u}}{\mathrm{d} y^{2}}=\frac{\mathrm{d} \tau}{\mathrm{d} y}=0$.

The velocity distribution in a laminar flows are $\boldsymbol{u}=\frac{\tau_{w}}{\mu}, \mu \frac{\mathrm{d} \boldsymbol{u}}{\mathrm{d} y}=$ const $=\tau_{w}$, $y$-distance in the vertical direction to the surface, $\mu$-viscosity, $\boldsymbol{u}$-velocity, $\tau_{w}$ friction. For the turbulent motion of turbulent shear the friction $\tau=-\rho \vec{u}^{\prime} v^{\prime}$ at the wall is zero. Reynolds equation $\frac{\mathrm{d}^{2} \boldsymbol{u}}{\mathrm{d} y^{2}}=\frac{\mathrm{d} \tau}{\mathrm{d} \boldsymbol{x}}$ If the shear stress given by the formula Prandtl $\tau=\rho l^{2}\left(\frac{\mathrm{d} \boldsymbol{u}}{\mathrm{d} \boldsymbol{x}}\right)^{2}=\tau_{w}, l=k y, k=$ const

$$
\boldsymbol{u}=\frac{1}{k \sqrt{\frac{\tau_{w}}{\rho}}} \ln y+C, C=\text { const }
$$

Thus, after obtaining the velocity profile by classic theory certain assumptions are used and did not solve of the Navier-Stokes equations and the boundary layer, but new equations by Prandtl. The initial equations are not satisfied on the external boundary or surface under no circumstances; no transition asymptotic solutions of problem for the semi-infinite plate to the solving the problem for infinite plate.

Historical (classical) formulation of conservation laws were based on a closed elementary volumes for exchange of the tangential component of the physical variables that led to the formulation of the conditions for the equilibrium of forces. Being open system, the elementary volume exchanged components physical quantities in all directions. Very importance is the role of the inertia centre [3]. In this paper we numerically investigate the influence of small perturbations of vertical velocity on the longitudinal velocity in the modified Falkner-Skan problem to reflect changes the influence of the angular momentum in the elementary volume. This modified task is difficult for the numerical solution, because the system of equations contain derivatives of the third order, being at the same time singular with small parameter near derivatives, as Navie-Stokes equations. We can find decision of the Korteweg-de Vries equation in many papers [17] [18]. The difficulties are associated with nonmonotonic solution of the equation. The second problem-is the interaction of two different parallel streams. The peculiarity of this task is singularity on two borders and the need to ensure continuity of the derivatives on the border interaction of flows. To study the de- 
lay in the kinetic theory is necessary due to the finiteness of the interaction time between the molecules and the definition of a derivative.

\section{Elements of General Modified Theory}

As follows from the previous work, taking into account the laws of conservation of angular momentum we have follows equations [1] [2] [3]

$$
\begin{gathered}
\frac{\partial \rho}{\partial t}+\frac{\partial \rho \boldsymbol{u}_{i}}{\partial \boldsymbol{x}_{i}}+\frac{\partial}{\partial \boldsymbol{x}_{i}}\left(\boldsymbol{x}_{i} \frac{\partial \rho \boldsymbol{u}_{i}}{\partial \boldsymbol{x}_{i}}\right)=0 \\
\frac{\partial \rho \boldsymbol{u}_{i}}{\partial t}+\frac{\partial}{\partial \boldsymbol{x}_{i}}\left(\rho \boldsymbol{u}_{i} \boldsymbol{u}_{j}+\boldsymbol{P}_{i j}+\boldsymbol{x}_{i} \frac{\partial \boldsymbol{P}_{i j}}{\partial \boldsymbol{x}_{i}}\right)-\frac{X_{i}}{m} \rho=0 \\
\frac{\partial}{\partial t} \rho\left(\frac{3}{2} R T+\frac{1}{2} \boldsymbol{u}^{2}\right)+\frac{\partial}{\partial \boldsymbol{x}_{j}}\left[\rho \boldsymbol{u}_{j}\left(\frac{3}{2} R T+\frac{1}{2} \boldsymbol{u}^{2}\right)+\boldsymbol{u}_{k} \boldsymbol{P}_{k j}+q_{j}\right]+\left[\boldsymbol{u}_{k} \boldsymbol{P}_{k j}+q_{j}\right] \\
+\frac{\partial}{\partial \boldsymbol{x}_{i}} \boldsymbol{x}_{i} \frac{\partial}{\partial \boldsymbol{x}_{j}}\left[\rho \boldsymbol{u}_{j}\left(\frac{3}{2} R T+\frac{1}{2} \boldsymbol{u}^{2}\right)+\boldsymbol{u}_{k} \boldsymbol{P}_{k j}+q_{j}\right]=0
\end{gathered}
$$

Here $t$-time, $\boldsymbol{x}_{i}$-coordinates, $\boldsymbol{u}_{i}$-speed, $\mu$-viscosity, $\rho$-density, $T$-temperature, $q$-thermal-flow, $\boldsymbol{P}_{i j}$-tensor of viscous pressure, $X-$ force. The system is complemented by the law of conservation law the angular momentum equation

$$
\frac{\partial \boldsymbol{r}}{\partial \boldsymbol{x}} \times \boldsymbol{p}_{x}+\frac{\partial \boldsymbol{r}}{\partial y} \times \boldsymbol{p}_{y}+\frac{\partial \boldsymbol{r}}{\partial \boldsymbol{z}} \times \boldsymbol{p}_{z}+\boldsymbol{x}_{j} \frac{\partial \boldsymbol{P}_{j}}{\partial \boldsymbol{x}}=M_{I}
$$

This equations were obtained from modified the Boltzmann equation [3].

$$
\frac{\mathrm{d} f}{\mathrm{~d} t}=\frac{\partial f}{\partial y}+c_{i} \cdot \frac{\partial f}{\partial \boldsymbol{x}_{i}}+c_{i} \cdot \frac{\partial}{\partial \boldsymbol{x}_{i}} r_{i} \frac{\partial f}{\partial r_{i}}-\frac{F}{m} \frac{\partial f}{\partial \boldsymbol{x}_{i}}=I .
$$

Here $I$-collision integral, $F$-force, $f$ is the one-particle distribution function, $\boldsymbol{x}$-coordinate of the point and according to definition of $f$ in element of physical volume $\mathrm{d} \boldsymbol{x}$ near the point $\boldsymbol{x}$ in moment $t$, probable number of molecules with velocity in element $\mathrm{d} c$ near the $c$ is $f(t, \boldsymbol{x}, c) \mathrm{d} c$, $t$ is the time, $\rho$ is the density, $x_{i}$ are the Cartesian coordinates of a particle, $X_{i}$ are the projections of a volume force, $q_{j}$ is the heat flow, $R$ is the gas constant and $T$ is a temperature. Another problem for the solving of the Boltzmann equation is the asymptotical methods. It is essential that selecting the local equilibrium distribution function $f^{0}$ as the basis in solution of the Boltzmann equation by the Chapman-Enskog method exploits macroscopic parameters in $f^{0}$ from the Euler equations [11]. Macroscopic parameters are determined by the Chapman-Enskog distribution function but used parameters from the Euler equations and tensor $\boldsymbol{P}$ is symmetrical. Formally in that way we have values (density, linear moment, energy) with mistake of the first order. As we mention this is the Hilbert paradox

$$
\int \varphi(\xi) f^{0} \mathrm{~d} \xi=\int \varphi(\xi) f \mathrm{~d} \xi=\beta
$$

Here $\beta$-macroscopic parameter, $\varphi(\xi)$-function. 


$$
\begin{gathered}
n(t, \boldsymbol{x})=\int f(t, \boldsymbol{x}, \boldsymbol{\xi}) \mathrm{d} \boldsymbol{\xi}, \boldsymbol{u}(t, \boldsymbol{x}) \frac{1}{n} \int \xi f(t, \boldsymbol{x}, \boldsymbol{\xi}) \mathrm{d} \boldsymbol{\xi}, \\
\boldsymbol{P}_{i j}=m \int c_{j} c_{i} f(t, \boldsymbol{x}, \boldsymbol{\xi}), q_{i}=\frac{m}{2} \int c^{2} c_{i} f(t, \boldsymbol{x}, \boldsymbol{\xi}) \mathrm{d} \boldsymbol{\xi}
\end{gathered}
$$

So for the equilibrium in collision integral

$$
f(t, \boldsymbol{x}, \boldsymbol{\xi}) \equiv f^{0}(t, \boldsymbol{x}, \boldsymbol{\xi})=n\left\{-\frac{m}{2 k T} c^{2}\right\}
$$

and for nonequilibrium distribution function

$$
f=f^{0}\left[1+\frac{\boldsymbol{p}_{i j} m}{2 p k T} c_{i} c_{j}-\frac{q_{i} m}{\boldsymbol{p} k T} c_{i}\left(1-\frac{m c^{2}}{5 k T}\right)\right]
$$

we have the same macroparameters in $f^{0}$. The nonequilibrium distribution function is such that integral of it contains only the integral of equilibrium function $f^{0}$ and gives containing in $f^{0}$ macroscopic parameters. The remaining summand gives null. We suggest to do another iteration for macroparameters selecting them from the Navier-Stokes equations. Elementary volume can be rotated around the axis of inertia or to be involved in the rotational movement. In both cases the density of the flow across the border changes by $\frac{\mathrm{d}(\rho \boldsymbol{u})}{\mathrm{d} r} \cdot\left(r^{\prime}-r\right)+\cdots \quad$ (Figure 1$)$ by rotation of the elementary volume. The contribution of other components is small, taking into account the smallness of the volume and the absence of rotation on axis.

\section{Falkner-Scan Task with Modified Boundary Conditions}

Consider the boundary layer with the forward motion of the cylinder at a speed at the outer edge $\left(U_{e}=a x^{m}\right)$ with input summand for angular momentum. This problem contains as a special case of the decision for the plate with a uniform external flow and interesting as an example of accelerated $(m>0)$ or delayed $(m<0)$ motion in the external flow $U_{e}$

$$
U_{e}=U_{\infty}+\varepsilon\left(\frac{\partial u}{\partial y}+\frac{\partial v}{\partial \boldsymbol{x}}\right)
$$

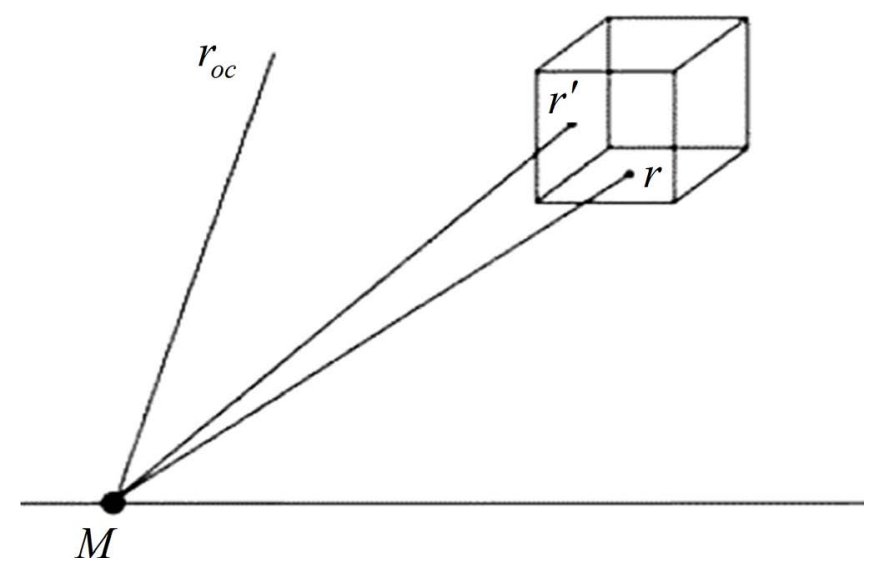

Figure 1. Element volume for density. 


$$
\begin{gathered}
\boldsymbol{u} \frac{\partial \boldsymbol{u}}{\partial y}+v \frac{\partial \boldsymbol{u}}{\partial y}=U_{e} \frac{\partial U_{e}}{\partial \boldsymbol{x}}+\frac{\partial}{\partial y}\left(\mu \frac{\partial \boldsymbol{u}}{\partial y}\right) \frac{\partial}{\partial y}\left(\mu y \frac{\partial \boldsymbol{u}^{2}}{\partial \boldsymbol{u}^{2}}\right) \\
\frac{\partial \boldsymbol{u}}{\partial \boldsymbol{x}}+\frac{\partial v}{\partial y}=0
\end{gathered}
$$

or

$$
\frac{\partial \boldsymbol{u}}{\partial \boldsymbol{x}}+\frac{\partial v}{\partial y}+\frac{\partial}{\partial y} y \frac{\partial v}{\partial y}=0
$$

with boundary conditions

$$
\boldsymbol{u}=0, v=0, \mu \frac{\partial u}{\partial y}=\tau_{w}=\alpha / \sqrt{x^{(m-1) / 2}} .
$$

$$
\boldsymbol{u}=U_{e}, y \rightarrow \infty, \boldsymbol{x}>0 ; \boldsymbol{u}=U_{e}, \boldsymbol{x}=0, U_{e}=a x^{m}, a=\text { const, } m=\text { const. }
$$

The numerical method was built. Managed to count 300 steps, then there was an increase the vertical velocity component. That requires another mathematical model. Solution of self-similar problem was carried out in [19].

At Figure 2 \& Figure 3, horizontal line-stretch coordinate

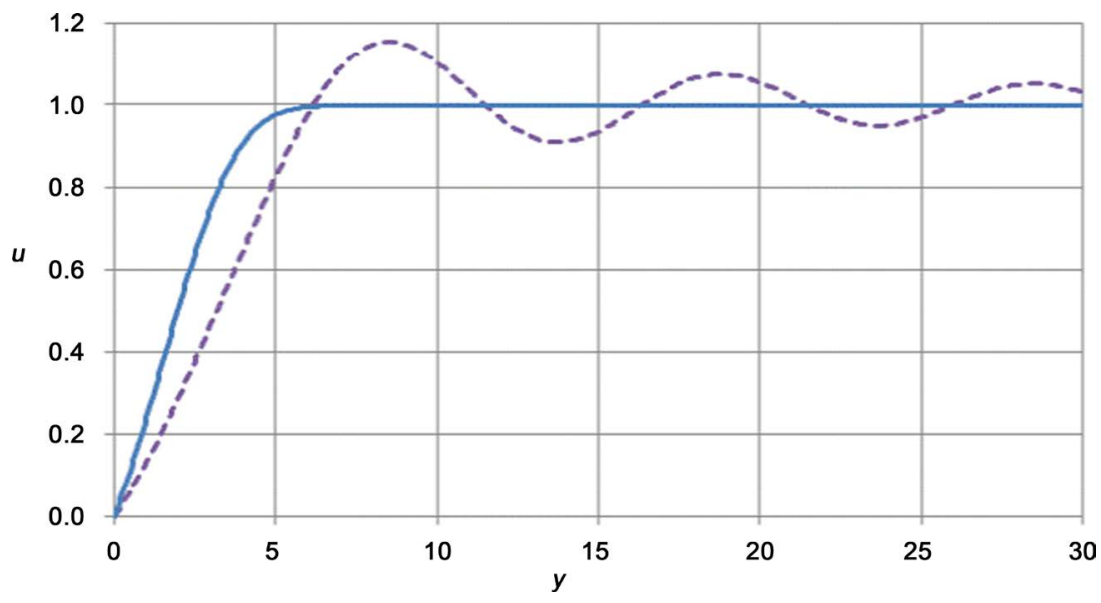

Figure 2. $m=-0.05 ; \tau_{w}=0.2202$ Profile $\Phi(\eta)$ along $\eta$ coordinate.

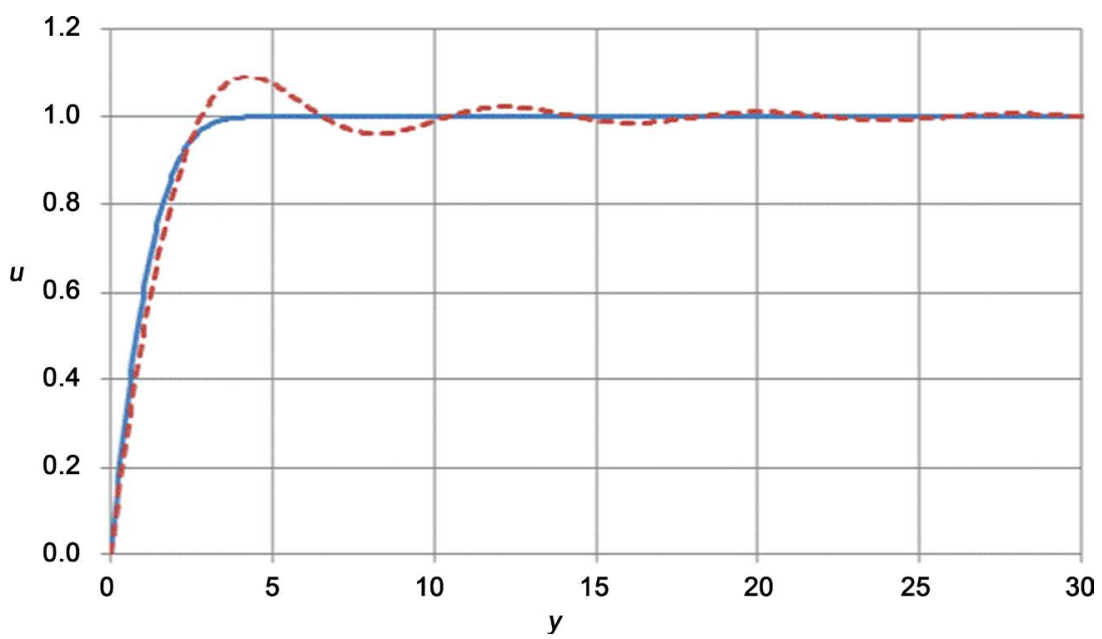

Figure 3. $m=0.33 ; \tau_{w}=0.7575$ Profile $\Phi(n)$ along $\eta$ coordinate. 


$$
\boldsymbol{u}=c x^{m} \Phi(n), \eta=\sqrt{\frac{c}{\mu}} y x^{(m-1) / 2}, v=\sqrt{\mu c} x^{\frac{m-1}{2}} V(n) .
$$

resulted by constant boundary conditions at the outer edge of the boundary layer.

Figures 2-7 illustrate the effects of the angular momentum on the velocity profiles in the boundary layer (Equation (8)). In the presence of the vortex perturbations around an axis $Z$, vortex structure is there inside the boundary layer. It is known [20] that vortex perturbations located for parallel flows, new structures are not allowed, but for turbulent flows there are various another structures within the boundary layer [13]-[23]. On vertical coordinate is $\boldsymbol{u}$, on horizontal coordinate is $y, \boldsymbol{u}$ and $v$.

Figure 8 produced the stream function for the latter option for $m=0$, $\tau_{w}=0.5, \varepsilon=0.005$.

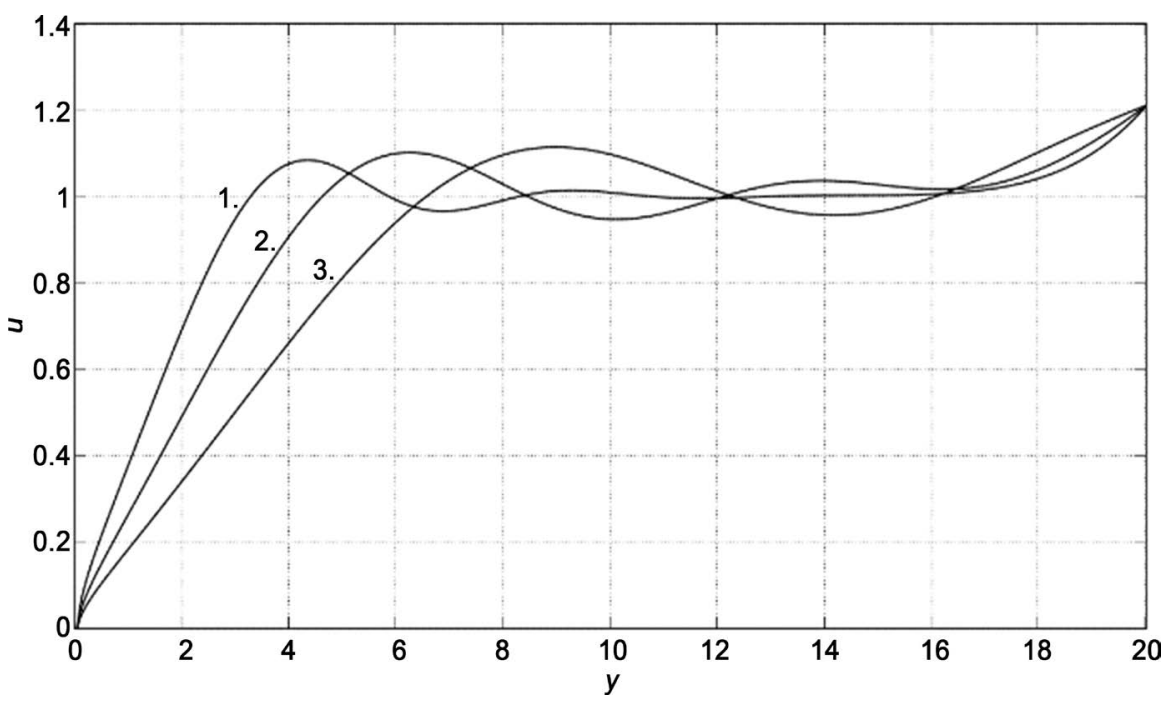

Figure 4. Profile of the horizontal velocity component along the vertical axis: $m=-0.04$, $\tau_{w}=0.5 ; 1 .-15^{\text {th }}$ layer, $2 .-30^{\text {th }}$ layer, $3 .-60^{\text {th }}$ layer

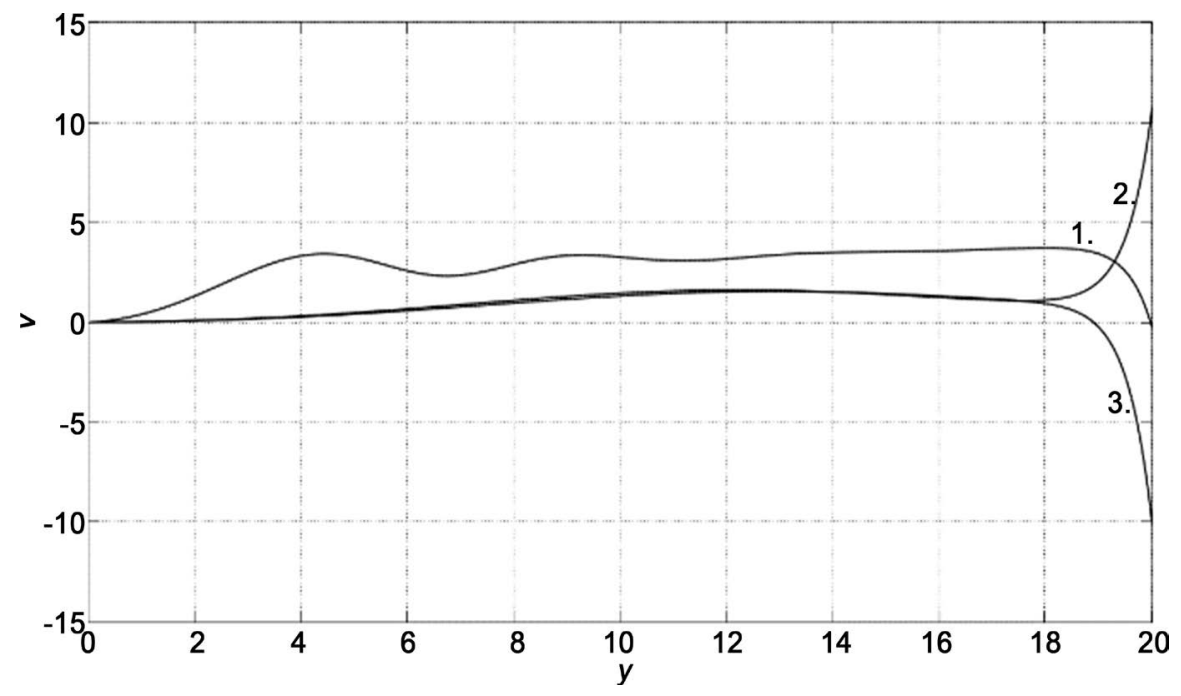

Figure 5. Profile $v, m=-0.04, \tau_{w}=0.5$. 


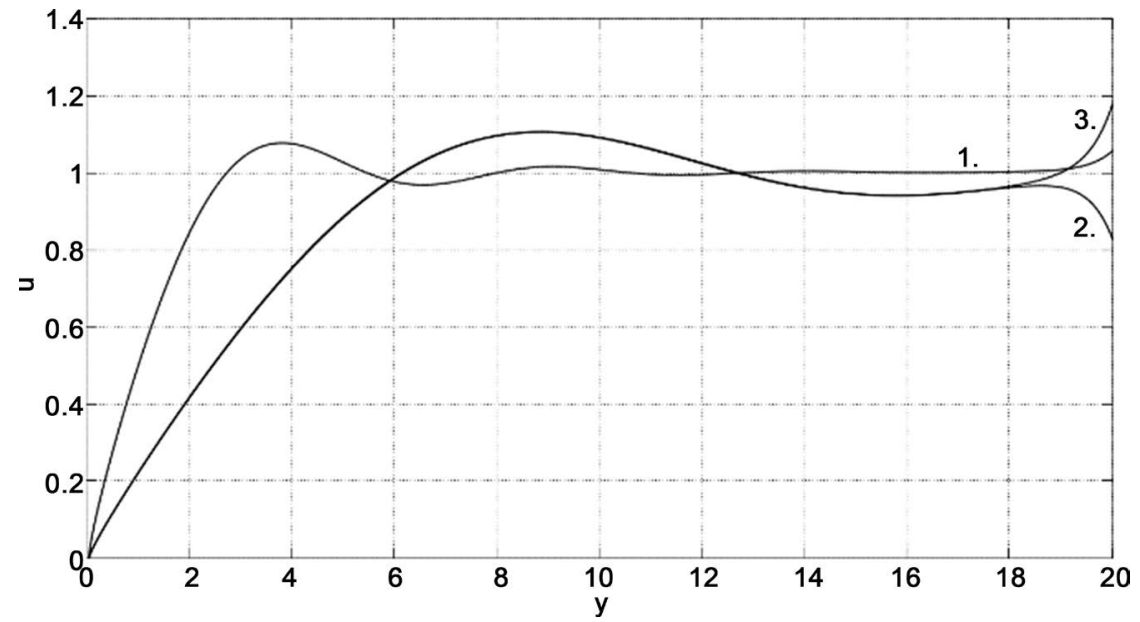

Figure 6. Profile of the horizontal velocity component along the vertical axis: $m=0$, $\tau_{w}=0.5 ; 1 .-15^{\text {th }}$ layer, $2 .-100^{\text {th }}$ layer, $3 .-101^{\text {st }}$ layer.

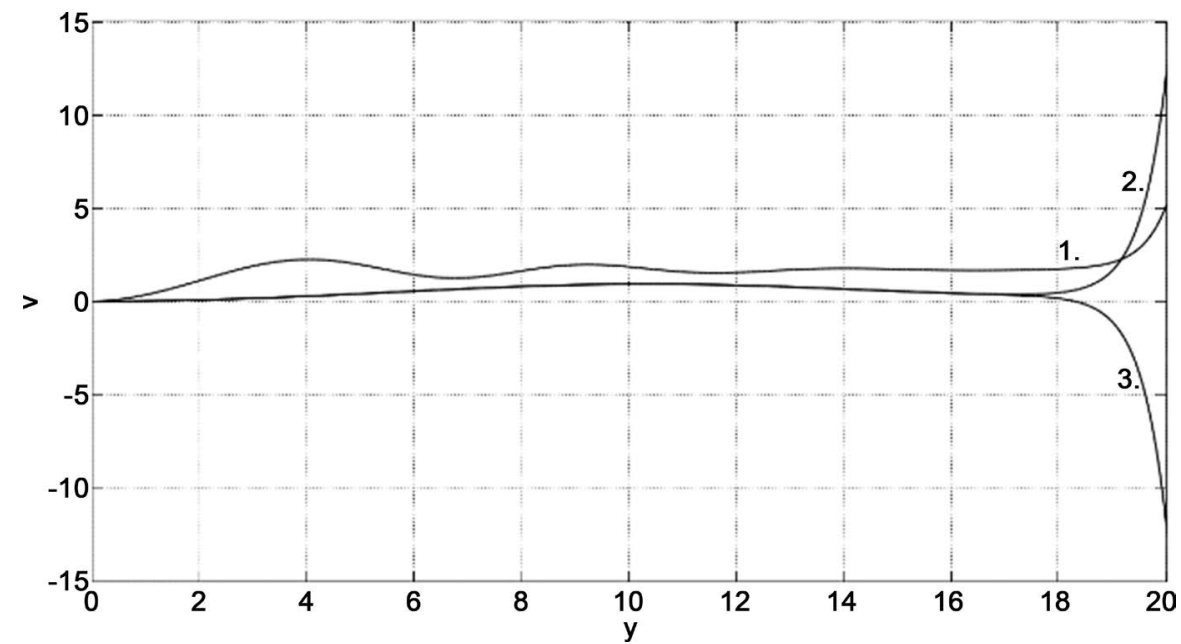

Figure 7. Profile $v, m=0, \tau_{w}=0.5$.

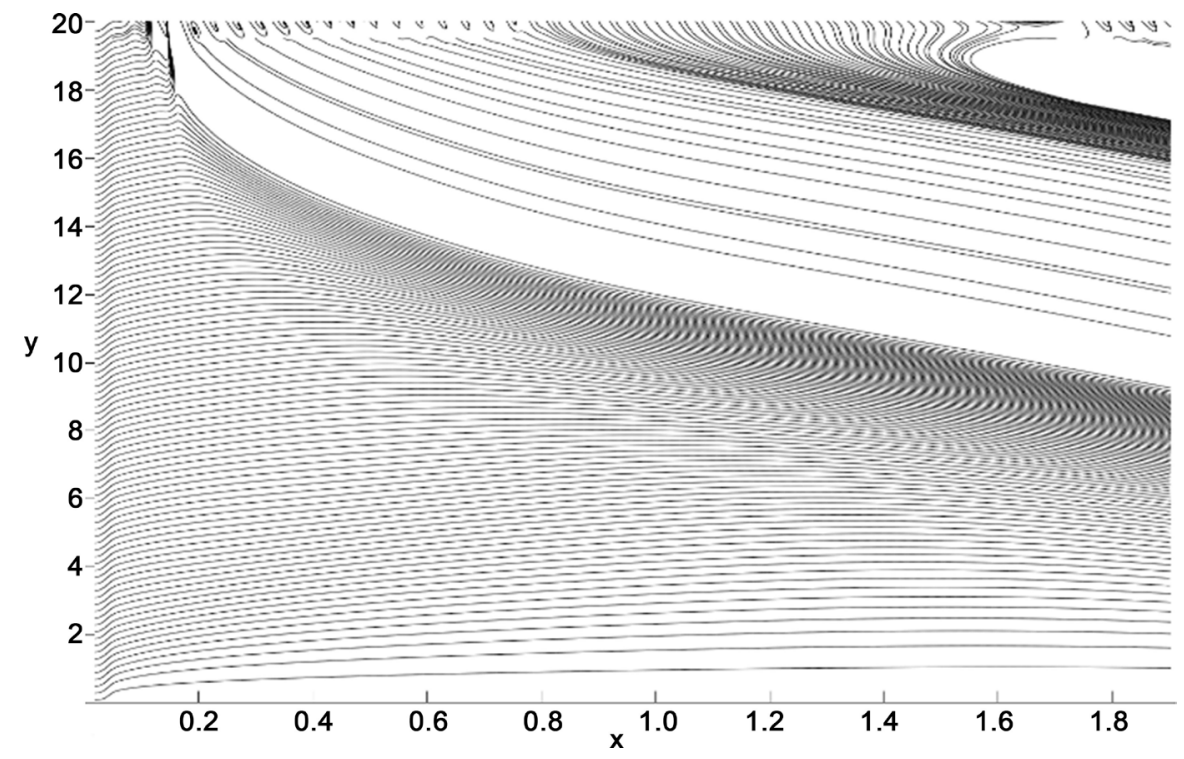

Figure 8. The stream function for the latter option for $m=0, \tau_{w}=0.5, \varepsilon=0.005$. 


\section{Free Layers of Mixing}

The simplest example of the transition layer between two homogeneous flows is diffusing vortex sheet. The fluid velocity in planes parallel vortex sheet is constant [24].

The schema the stream is produced on Figure 9. Install the free mixing layer, by definition, varies along the coordinate $\boldsymbol{x}, \boldsymbol{u}$, hence the boundary layer equations are applicable. Homogeneous flow of the same fluid moves in parallel increasing $x$ coordinate at different speeds $U 1$ and $U 2$ (Figure 1). The problem is self-similar, singular and necessary to apply non-standard method of solution. We divide the area into two parts, the initial value of the friction is given on line 0 . The problem is resolved in every part for the second derivative of the current function. The velocity function is obtained by integrating the function. The iterative process is will be done until convergence.

$$
\boldsymbol{u} \frac{\partial \boldsymbol{u}}{\partial \boldsymbol{x}}+v \frac{\partial \boldsymbol{u}}{\partial y}=U_{e} \frac{\partial U_{e}}{\partial \boldsymbol{x}}+\frac{\partial}{\partial y}\left(\mu \frac{\partial \boldsymbol{u}}{\partial y}\right)+\frac{\partial}{\partial y}\left(\mu y \frac{\partial^{2} \boldsymbol{u}}{\partial y^{2}}\right), \frac{\partial \boldsymbol{u}}{\partial \boldsymbol{x}}+\frac{\partial v}{\partial y}=0
$$

After a dimensionless functions $U_{e}$ we have

$$
\begin{aligned}
& -1 / 2 \mathrm{D} \Phi \frac{\mathrm{d} \Phi}{\mathrm{d} \eta \eta}+V \frac{\mathrm{d} \Phi}{\mathrm{d}}=\mu \frac{\mathrm{d}^{2} \Phi}{\mathrm{d}^{2}}+\frac{\mathrm{d}}{\mathrm{d} \eta}\left(\mu \mathrm{n} \frac{\mathrm{d}^{2} \Phi}{\mathrm{d}^{2}}\right) \\
& -1 / 2 \mathrm{n} \frac{\mathrm{d} \Phi}{\mathrm{d \eta} \eta}+\frac{\mathrm{d} V}{\mathrm{~d}}=0
\end{aligned}
$$

SubstitutingVwe getthe equation

$$
\mu \frac{\mathrm{d}}{\mathrm{d} \eta}\left(\eta \frac{\mathrm{d}^{3} \Psi}{\mathrm{dn}^{3}}\right)+\mu \frac{\mathrm{d}^{3} \Psi}{\mathrm{dn}^{3}}+2 \Psi \frac{\mathrm{d}^{2} \Psi}{\mathrm{dn}^{2}}=0
$$

Here $\Psi^{*}=\sqrt{\mu / U_{1}} \Psi, y_{1}=1 / 2 \sqrt{\mu / U_{1}} \eta$.

Then we have

$$
2 n \Psi^{\prime \prime \prime \prime}+2 \Psi^{\prime \prime \prime}+\Psi \Psi^{\prime \prime}=0
$$

with boundary conditions

$$
\Psi(0)=0, \Psi^{\prime}(0)=1-a, \Psi^{\prime \prime}(0+)=\Psi^{\prime \prime}(0-), \Psi(-\infty)=a
$$

Analytical solutions have not been found. The numerical result is on Figure 10.

The friction is greater than for classic case.

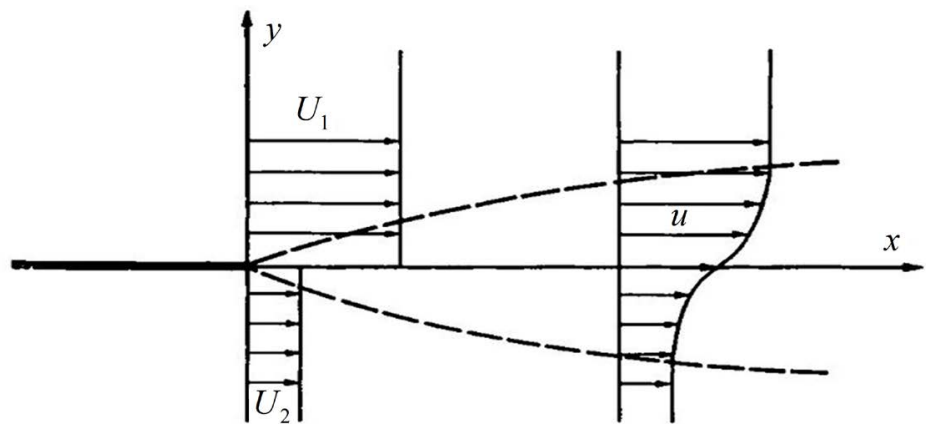

Figure 9. Set the transition layer between two parallel streams, the contacting at $x=0$. 


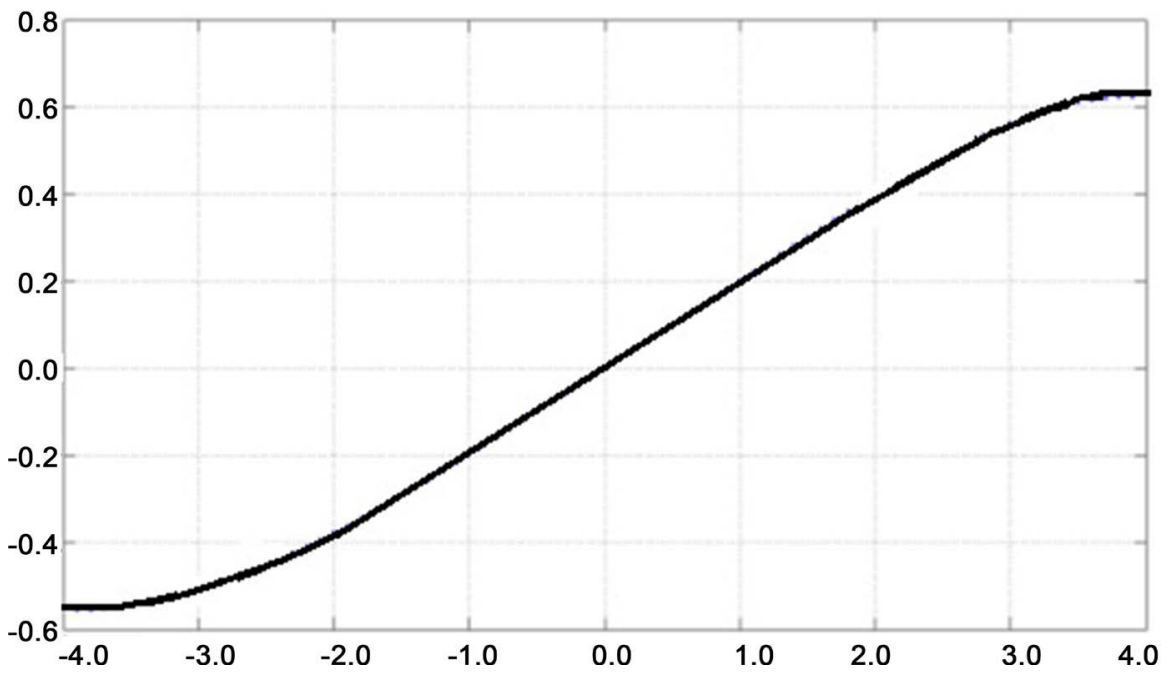

Figure 10. The calculation results for $a=0.4$.

\section{Exact Solution for Kinetic Theory: The Barometric Boltzmann Formula}

Gas is at stationary condition in field of force which has potential $\varphi$ (this is analogy tasks from [10]):

As before solution we shall be look for as $f=A(\boldsymbol{x}) \mathrm{e}^{-B(x) \xi^{2}}$. We receive the old result $B=$ Const. For $A(x)$ we have equation

$$
\frac{\mathrm{d} A}{\mathrm{~d} \boldsymbol{x}}+\frac{\mathrm{d}}{\mathrm{d} \boldsymbol{x}_{i}} \boldsymbol{x}_{i} \frac{\mathrm{d} A}{\mathrm{~d} \boldsymbol{x}_{i}}+2 \frac{A \cdot B}{m} \frac{\partial \varphi}{\partial \boldsymbol{x}_{i}}=0 .
$$

We have old result that is common for one-dimension tasks:

$$
f=n_{0}\left(\frac{m}{2 \pi k T}\right)^{3 / 2} \mathrm{e}^{-\frac{\varphi}{k T}} \mathrm{e}^{-\frac{m}{2 k T} \xi^{2}} .
$$

General local-Makswell distribution is

$$
f=n\left(\frac{m}{2 \pi k T}\right)^{3 / 2} \exp \left\{-\frac{m}{2 k T} c^{2}\right\}, c=\boldsymbol{\xi}-\boldsymbol{u} .
$$

Modification the Boltzmann equation is

$$
\boldsymbol{\xi}_{i} \frac{\partial f}{\partial \boldsymbol{x}_{i}}+\boldsymbol{\xi}_{i} \frac{\partial}{\partial \boldsymbol{x}_{i}} \boldsymbol{x}_{i} \frac{\partial f}{\partial \boldsymbol{x}_{i}}-g_{i} \frac{\partial f}{\partial \boldsymbol{\xi}_{i}}=J(f, f) .
$$

$g=X / m$ - acceleration of molecules.

Let local-Makswell solution of the equation will be considered as in old algorithm

$$
\ln f=\gamma_{0}+\gamma_{i} \xi_{i}+\gamma_{4} \xi^{2}
$$

Then we receive old equation and the changing

$$
\begin{gathered}
\frac{\partial \gamma_{0}}{\partial t}+g_{i} \gamma_{i}=0 \\
\frac{\partial \gamma_{i}}{\partial t}+2 g_{i} \gamma_{4}+\frac{\partial \gamma_{0}}{\partial \boldsymbol{x}_{i}}+\frac{\partial \gamma_{0}}{\partial \boldsymbol{x}_{i}}+\frac{1}{2} \boldsymbol{x}_{i} \frac{\partial \gamma_{0}^{2}}{\partial \boldsymbol{x}_{i}^{2}}+\frac{\partial}{\partial \boldsymbol{x}_{i}} \boldsymbol{x}_{i} \frac{\partial \gamma_{0}}{\partial \boldsymbol{x}_{i}}=0,
\end{gathered}
$$




$$
\begin{aligned}
& \frac{\partial \gamma_{4}}{\partial t} \delta_{i j}+\frac{1}{2}\left(\frac{\partial \gamma_{i}}{\partial \boldsymbol{x}_{j}}+\frac{\partial \gamma_{j}}{\partial \boldsymbol{x}_{i}}\right)+\frac{1}{2}\left(\frac{\partial \gamma_{i}}{\partial \boldsymbol{x}_{j}}+\frac{\partial \gamma_{j}}{\partial \boldsymbol{x}_{i}}\right)+\frac{1}{2} * \frac{1}{2}\left(\boldsymbol{x}_{i}+\boldsymbol{x}_{j}\right)\left(\frac{\partial \gamma_{i}}{\partial \boldsymbol{x}_{j}}+\frac{\partial \gamma_{j}}{\partial \boldsymbol{x}_{i}}\right) \frac{\partial \gamma_{0}}{\partial \boldsymbol{x}_{i}} \\
& +\frac{1}{2}\left(\boldsymbol{x}_{i}+\boldsymbol{x}_{j}\right) \frac{1}{2}\left(\frac{\partial}{\partial \boldsymbol{x}_{j}}\left(\frac{\partial \gamma_{i}}{\partial \boldsymbol{x}_{j}}+\frac{\partial \gamma_{j}}{\partial \boldsymbol{x}_{i}}\right)+\frac{\partial}{\partial \boldsymbol{x}_{i}}\left(\frac{\partial \gamma_{i}}{\partial \boldsymbol{x}_{j}}+\frac{\partial \gamma_{j}}{\partial \boldsymbol{x}_{i}}\right)\right)=0,
\end{aligned}
$$

As before

$$
\frac{\partial \gamma_{4}}{\partial \boldsymbol{x}_{i}}=0, T=\text { const. }
$$

As the result we shall receive modified gas-dynamic equations but without viscosity and thermal conductivity

\section{The Role of Angular Momentum and Delay}

In general case three positions are important for understanding the causes of modification the theory [1] [2] [3]. Another type of including angular momentum is contained in [6].

1) It is necessary to take for equilibrium conditions more general conditioncondition of angular momentum, although equilibrium of force is needed to retain but with non-symmetric stress tensor

2) Replacement of velocity decomposition near point of elementary volume to the decomposition near axis of inertia

3) Delay

For kinetic theory is need to investigate the question what is measured in experiment: the role of delay: at present moment or for middle results. If in experiment middle results are measured then it is essential to choose time and scale.

If the time is in coordination then the delay is no need to take into consideration besides the cases for relaxation time where we need to coordinate the delay. Then the Boltzmann equation is

$$
\begin{gathered}
\frac{\mathrm{d} f}{\mathrm{~d} t}=\frac{\partial f}{\partial t}+c_{i} \cdot \frac{\partial f}{\partial r_{i}}+c_{i} \cdot \frac{\partial}{\partial r_{i}} r_{i} \frac{\partial f}{\partial r_{i}}-\frac{F}{m} \frac{\partial f}{\partial c_{i}}=I \\
\frac{\mathrm{d} f}{\mathrm{~d} t}=\frac{\partial f}{\partial t}+\bar{\tau} \frac{\partial^{2} f}{\partial^{2} t}+c_{i} \cdot \frac{\partial f}{\partial r_{i}}+c_{i} \cdot \frac{\partial}{\partial r_{i}} r_{i} \frac{\partial f}{\partial r_{i}}-\frac{F}{m} \frac{\partial f}{\partial c_{i}}=I \\
\Delta^{-}=\mathrm{d} t \mathrm{~d} \boldsymbol{x} \mathrm{d} \xi f(t, \boldsymbol{x}, \boldsymbol{\xi}) \int\left[f_{1}(t, \boldsymbol{x}, \boldsymbol{\xi})+O\left(\Delta t \boldsymbol{\xi} \frac{\partial f_{1}}{\partial x}\right)\right] g b \mathrm{~d} b \mathrm{~d} \varepsilon \mathrm{d} \xi_{1} \\
\left.\Delta^{+}=\mathrm{d} t \mathrm{~d} \boldsymbol{x} \mathrm{d} \boldsymbol{\xi}^{\prime}\right]\left[f\left(t, \boldsymbol{x}, \boldsymbol{\xi}^{\prime}\right) f\left(t, \boldsymbol{x}, \boldsymbol{\xi}_{1}^{\prime}\right)+O\left(\Delta t \boldsymbol{\xi}_{\frac{\partial}{\partial x}}\right)\right] g^{\prime} b^{\prime} \mathrm{d} \varepsilon^{\prime} \mathrm{d} \boldsymbol{\xi}_{1} \\
I=\Delta^{-}-\Delta^{+} \\
\frac{\partial f}{\partial t} \leftrightarrow \frac{\partial f}{\partial t}+\bar{\tau} \frac{\partial^{2} f}{\partial^{2} t} \\
f^{\prime}\left(t, \boldsymbol{x}, \boldsymbol{\xi}^{\prime}\right) \leftrightarrow f^{\prime}\left(t, \boldsymbol{x}, \boldsymbol{\xi}^{\prime}\right) \\
f(t, \boldsymbol{x}, \boldsymbol{\xi}) \leftrightarrow f(t+\bar{\tau}, \boldsymbol{x}+\bar{\lambda}, \boldsymbol{\xi}) \leftrightarrow f(t, \boldsymbol{x}, \boldsymbol{\xi})+\bar{\tau} \frac{\partial f}{\partial t}+\bar{\lambda} \frac{\partial f}{\partial x}+\cdots
\end{gathered}
$$




$$
f_{1}\left(t, \boldsymbol{x}, \boldsymbol{\xi}_{1}\right) \leftrightarrow f_{1}\left(t+\bar{\tau}, \boldsymbol{x}+\bar{\lambda}, \boldsymbol{\xi}_{1}\right) \leftrightarrow f_{1}\left(t, \boldsymbol{x}, \boldsymbol{\xi}_{1}\right)+\bar{\tau} \frac{\partial f_{1}}{\partial t}+\bar{\lambda} \frac{\partial f_{1}}{\partial \boldsymbol{x}}+\cdots
$$

In the formulas selected averages, although one can select to individual speeds and consider all their sums. Similarly, calculated values with the molecule that is flying during the free path, the mean free path of the molecules and the travel time to and after the collision can be different (may be different values of the incident and impinging molecules (with index one))

$$
\begin{aligned}
& f f_{1}-f f_{1}^{\prime} \leftrightarrow f f_{1}-f f_{1}^{\prime}+\tau \frac{\partial f}{\partial t} f_{1}+\tau_{1} f \frac{\partial f_{1}}{\partial t}+\lambda \frac{\partial f}{\partial \boldsymbol{x}} f_{1}+\lambda_{1} f \frac{\partial f_{1}}{\partial \boldsymbol{x}} \\
& +\cdots-\tau^{\prime} \frac{\partial f^{\prime}}{\partial t} f_{1}^{\prime}--\tau_{1}^{\prime} f \frac{\partial f_{1}^{\prime}}{\partial t}-\cdots-\lambda^{\prime} \frac{\partial f^{\prime}}{\partial \boldsymbol{x}} f_{1}^{\prime}-\lambda_{1}^{\prime} \frac{\partial f_{1}^{\prime}}{\partial \boldsymbol{x}} f^{\prime}-\cdots
\end{aligned}
$$

In general, this formula should be writing in this form, but for small gradients of simple gas can be write to single time and single long of the run. However, for structural gas, for example, at altitudes above $120 \mathrm{~km}$ the free time of run on three Mach numbers, i.e. lag time $10^{-8} \mathrm{c}$ and more that can be comparable with the relaxation time. In fact, the expression can be simplified by considering the order of magnitude. Then we have

$$
\begin{aligned}
& f f_{1}-f f_{1}^{\prime} \leftrightarrow f f_{1}-f f_{1}^{\prime}+\tau \frac{\partial f^{0}}{\partial t} f_{1}^{0}+\tau_{1} f^{0} \frac{\partial f_{1}^{0}}{\partial t}+\lambda \frac{\partial f^{0}}{\partial \boldsymbol{x}} f_{1}^{0}+\lambda_{1} \frac{\partial f_{1}}{\partial \boldsymbol{x}} f^{0} \\
& +\cdots-\tau^{\prime} \frac{\partial f^{\prime 0}}{\partial t} f_{1}^{\prime 0}-\tau_{1}^{\prime} f^{0} \frac{\partial f_{1}^{\prime 0}}{\partial t}-\cdots-\lambda^{\prime} \frac{\partial f^{\prime 0}}{\partial \boldsymbol{x}} f_{1}^{\prime 0}-\lambda_{1}^{\prime} \frac{\partial f_{1}^{\prime 0}}{\partial \boldsymbol{x}} f^{\prime}-\cdots
\end{aligned}
$$

Then the integrals are computed and we obtain the corresponding kernel of the Navier-Stokes equations. For small and medium gradients free time is single and single mean free path. But significant differences are in the interaction of gases with different properties. Will be discussed the situation when we have the density for the two interaction elementary volume which are much different. Another situation when properties of molecules are close. In order to evaluate we are using single mean free time and single mean free path. The density after collision is counted to equal to density of impinging of particles.

The preceding value of right part will be $\left(n \gg n_{1}\right)$

$$
\left(\tau-\frac{n}{n_{1}} \tau\right) \frac{\partial f^{0}}{\partial t} f_{1}^{0}+\left(\frac{n}{n_{1}} \tau-\frac{n}{n_{1}} \tau\right) f^{0} \frac{\partial f_{1}^{0}}{\partial t}+\left(\lambda-\frac{\xi^{\prime}}{\xi} \lambda\right) \frac{\partial f^{0}}{\partial \boldsymbol{x}} f_{1}^{0}+\left(\frac{n_{1}}{n} \lambda-\frac{n}{n_{1}} \lambda\right) \frac{\partial f_{1}}{\partial \boldsymbol{x}} f^{0}
$$

Derivative on $\boldsymbol{x}$ is given us self diffusion, termo-diffusion and baro-diffusion about which S. V. Vallander told. Direct influence on delay the first item has $C \frac{n}{n_{1}} \tau \frac{\partial f^{0}}{\partial t} f_{1}^{0}$. If count up that $n \gg$ we shall receive the main item $\frac{n}{n_{1}} \tau \frac{\partial f^{0}}{\partial t}$. It is additive core of the modified Boltzmann equation and the Navier-Stokes equations. It is necessary to take into the attention for rarefied gas of complicated molecules with time relaxation is near time between collisions.

\section{Conclusion}

Influence of the angular momentum is investigated and, as consequence, nonsymmetry of stress tensor is received for elementary volume. The role of delay is 
investigated in kinetic theory. It shows the influence of these effects in continuum mechanics and kinetic theory, and some the numerical results are demonstrated (the Falkner-Skan problem). The striped structure was received. Vertical component of velocity plays more roles if the angular momentum is taken into account. The perturbations on upper of boundary layer form the vortex near the surface. The exact solution of kinetic theory that is well known in classic is considered.

\section{References}

[1] Prozorova, E.V. (2014) Influence of Mathematical Models in Mechanics. Problems of Nonlinear Analysis in Engineering Systems, 20, 78-86.

[2] Prozorova, E.V. (2012) The Effect of Dispersion in Nonequilibrium Continuum Mechanics Problems Environment. Physico-Chemical Kinetics in Gas Dynamics, 13. (In Russian) http://www.chemphys.edu.ru/pdf/2012-10-30-001.pdf

[3] Prozorova, E.V. (2014) Influence of the Delay and Dispersion In mechanics. Journal of Modern Physics, 5, 1796-1805. https://doi.org/10.4236/jmp.2014.516177

[4] Cosserat, E. and Cosserat, F. (1909) Theories des corps deformables. Hermann, Paris.

[5] Efendiev, Y., Galvis, J. and Hou, T. (2013) Generalized Multiscale Finite Element Method. Journal of Computational Physics, V, 116-135.

[6] Bulanov, E.A. (2012) The Momentum Tension on Mechanics of Solid, Free Flowing and Liquid Medium. College Book, Moment.

[7] Elizarova, T.G. (2007) Guasi-Gasdynamic Equations and Numerical Methods for Viscous Flow Simulation. M.:Scientific Word, 352.

[8] Cercignani, C. (1969) Mathematical Methods in Kinetic Theory. Macmillan, 148 p. https://doi.org/10.1007/978-1-4899-5409-1

[9] Ferziger, J.H. and Kaper, H.G. (1972) Mathematical Theory of Transport Processes in Gases. North-Holland Publishing Company, Amsterdam, London.

[10] Hirschfelder, J.O., Curtiss, C.F. and Bird, R.B. (1954) The Molecular Theory of Gases and Liquids. New York.

[11] Kogan, M.N. (1967) The Dynamics of the Rarefied Gases. M.: Nauka. (In Russian)

[12] Loyotinskiy, L.G. (1970) Mechanics of Fluids and Gas. M.: Nauka. (In Russian)

[13] Kameda, Y., Yoshino, J. and Ishihara, T. (2008) Examination of Kolmogorov's 4/5 Law by High-Resolution Direct Numerical Simulation Data of Turbulence. Journal of the Physical Society of Japan, 77, Article ID: 064401. https://doi.org/10.1143/JPSJ.77.064401

[14] Adamian, D.Y., Strelets, M.K. and Travin, A.K. (2011) An Efficient Method of Synthetic Turbulence Generation at LES Inflow in Zonal RANS-LES Approaches to Computation Flows. Mathematical Modeling, 23. (In Russian)

[15] Natrajan, V.K., Wu, Y. and Christensen, K.T. (2007) Spatial Signatures of Retrograde Spanwise Vortices in Wall Turbulence. Journal of Fluid Mechanics, 574, 155167. https://doi.org/10.1017/S0022112006003788

[16] Guala, M., Liberzon, A., Tsinober, A. and Kinzelbach, W. (2007) An Experimental Investigation on Lagrangian Correlations of Small-Stale Turbulence at Law Reynolds Number. Journal of Fluid Mechanics, 574, 405-427. https://doi.org/10.1017/S0022112006004204

[17] Rashid, A. (2007) Numerical Solution of Korteweg-de Vries Equation by the Fourier 
Pseudospectral Method. Bulletin of the Belgian Mathematical Society-Simon Stevin, 14, 709-721.

[18] Popov, S.P. (2015) Numerical Analysis of Soliton Solutions of the Modified Korteweg-de Vries-Sine-Gordon Equation. Computational Mathematics and Mathematical Physics, 55, 437-446.

[19] Kononenko, V.A., Prozorova, E.V. and Shishkin, A.V. (2009) Influence Dispersion for Gas Mechanics with Great Gradients. 27 th International Symposium on Shock Waves, St. Petersburg, 19-24 July 2009, 406-407.

[20] Katasonov, M.M., Kozlov, V.V., Nikitin, N.V. and Sboev, D.C. (2015) Beginnings and Development of Localization Indignation in Circle Tube and Boundary Layer. Educational Text-Book, State University, Novosibirsk.

[21] Babkin, V.A. and Nicholas, V.N. (2009) Turbulent Flow in Acircular Tubeanda Flat channel and model Mesoscale Turbulence. Engineering Physics Magazine, 84, 5672.

[22] Elsinga, G.E., Adrian, R.J., Van Oudhensden, B.W. and Scarano, F. (2010) ThreeDimensional Vortex Organization in a High-Reynolds-Number Supersonic Turbulent Boundary Layer. Journal of Fluid Mechanics, 644, 35-60. https://doi.org/10.1017/S0022112009992047

[23] Priezjev, N.V. and Trouan, S.M. (2006) Influence of Periodic Wall Roughness on Slip Behaviour at Liquid/Solid Interfaces: Molecular-Scale Simulations versus Continuum Predictions. Journal of Fluid Mechanics, 554, 25-47.

[24] Batchelor, G.K. (1970) An Introduction to Fluid Dynamics. Cambridge of University Press, Cambridge.

Submit or recommend next manuscript to SCIRP and we will provide best service for you:

Accepting pre-submission inquiries through Email, Facebook, LinkedIn, Twitter, etc. A wide selection of journals (inclusive of 9 subjects, more than 200 journals)

Providing 24-hour high-quality service

User-friendly online submission system

Fair and swift peer-review system

Efficient typesetting and proofreading procedure

Display of the result of downloads and visits, as well as the number of cited articles

Maximum dissemination of your research work

Submit your manuscript at: http://papersubmission.scirp.org/

Or contact jamp@scirp.org 